\title{
Approximating the Numéraire Portfolio by Naive Diversification
}

October 20, 2011

\begin{abstract}
Estimation theory has shown, due to the limited estimation window available for real asset data, the sample based Markowitz mean-variance approach produces unreliable weights which fluctuate substantially over time. This paper proposes an alternate approach to portfolio optimization, being the use of naive diversification to approximate the numéraire portfolio. The numéraire portfolio is the strictly positive portfolio that, when used as benchmark, makes all benchmarked nonnegative portfolios either mean decreasing or trendless. Furthermore, it maximizes expected logarithmic utility and outperforms any other strictly positive portfolio in the long run. The paper proves for a well-securitized market that the naive equal value weighted portfolio converges to the numéraire portfolio when the number of constituents tends to infinity. This result is model independent and, therefore, very robust. The systematic construction of diversified stock indices by naive diversification from real data is demonstrated. Even when taking transaction costs into account, these indices significantly outperform the corresponding market capitalization weighted indices in the long run, indicating empirically their asymptotic proximity to the numéraire portfolio. Finally, in time of financial crisis, a large equi-weighted fund carrying the investments of major pension funds and insurance companies would provide important liquidity. It would not only dampen the drawdown of a crisis but would also moderate the excesses of an asset price bubble.
\end{abstract}

JEL Classification: G10, G13

2000 Mathematics Subject Classification: 65C30, 60H35, 60H30

Key words and phrases: Numéraire portfolio, growth optimal portfolio, Kelly portfolio, naive diversification, equi-weighted index, market capitalization weighted index, liquidity, Sharpe ratio. 


\section{Introduction}

The ground breaking work of Markowitz (1952) heralded considerable advances in asset allocation by deriving a methodology for allocating wealth across risky investments when investors base their strategies on the means, variances and covariances of asset returns. The resulting Markowitz mean-variance approach to investing has been generalized in many ways; see Campbell and Viceira (2002) for a survey. In each case the practical implementation of an optimal investment strategy depends crucially on the estimation of expected asset returns. Unfortunately, due to the rather limited estimation window available for real asset data, implementations of the sample based Markowitz approach and its extensions perform poorly out-of-sample; see for instance Best and Grauer (1991) and Litterman and the Quantitative Research Group (2003). A recent paper by DeMiguel, Garlappi and Uppal (2009) evaluated in detail the out-of-sample performance of sample based mean-variance asset allocation strategies, relative to the naive equal value weighted counterpart. The outcome being that of the 14 estimation procedures evaluated across seven empirical datasets, no strategy consistently outperformed the naive equal value weighted strategy in terms of Sharpe ratio and other common performance criteria. The authors concluded that the theoretical gain from classical sample based mean-variance asset allocation is more than offset by estimation errors. They demonstrated that, for a portfolio of 50 assets, the estimation window needed to outperform the naive equal value weighted portfolio is about 500 years. It is even longer for portfolios with a greater number of securities. This suggests that in the real market, consisting of several tens of thousands of stocks, there is no way of successfully implementing the classical sample-based mean-variance approach. The contribution of the current paper is to provide an explanation and further empirical support for the aforementioned findings of DeMiguel, Garlappi and Uppal (2009), as well as a robust methodology to approximate the numéraire portfolio (NP), which is the strictly positive portfolio that, when used as benchmark makes all benchmarked nonnegative portfolios either mean decreasing or trendless. More precisely, it makes any benchmarked nonnegative self-financing portfolio a supermartingale; see Shiryaev (1984) and (3.3).

The basic assumption of the current paper is concerned with the existence of the NP, which has been studied in many papers including Long (1990), BajeuxBesnainou and Portait (1997), Becherer (2001), Platen (2002), Bühlmann and Platen (2003), Platen (2006), Platen and Heath (2006), Karatzas and Kardaras (2007) and Kardaras and Platen (2008). For the NP its current benchmarked value is greater than or equal to its future expected benchmarked values. The NP also appears in the literature as the Kelly portfolio or growth optimal portfolio, see Kelly (1956), which maximizes the expected logarithmic utility from terminal wealth. There is an extensive literature on the growth optimal or Kelly portfolio, which includes Markowitz (1976), Latané (1959), Breiman (1961), Hakansson (1971), Thorp (1972), Merton (1973), Rubinstein (1976), Cover (1991), Luen- 


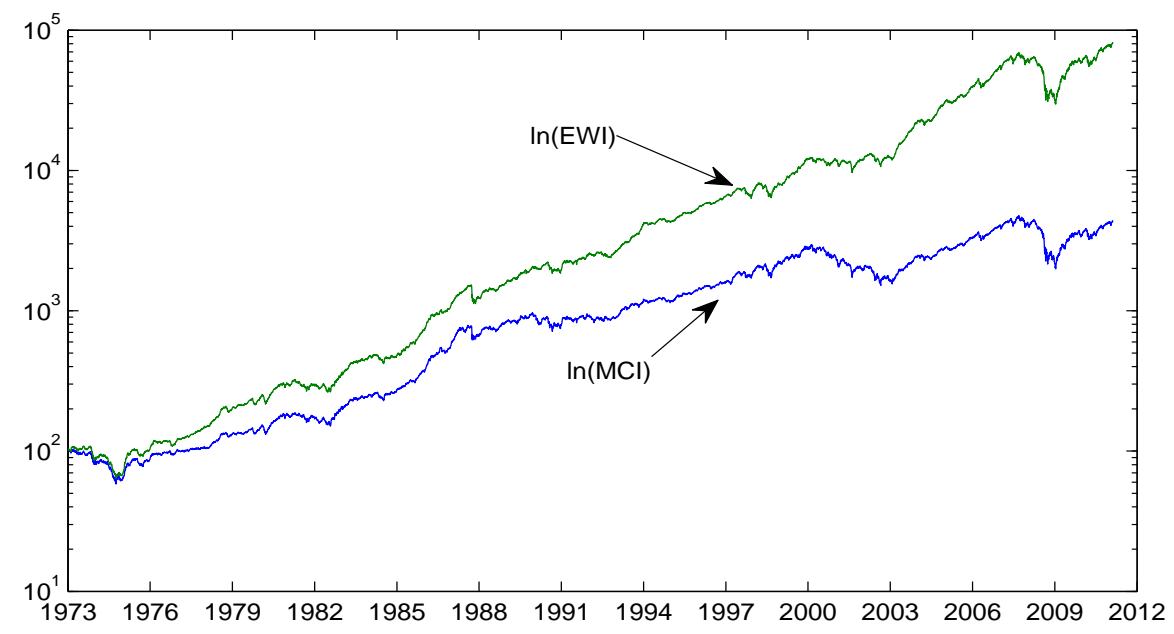

Figure 1.1: Logarithms of a market capitalization weighted index (MCI) and an equi-weighted index (EWI) under $40 \mathrm{bp}$ transaction costs.

berger (1998), Ziemba and Mulvey (1998), Browne (1999), Stutzer (2000), Platen and Heath (2006) and MacLean et.al. (2011). In the long run the NP outperforms pathwise any other nonnegative portfolio; see e.g. Platen (2004). This is a model independent and fascinating property, which makes the NP an ideal candidate for long term asset allocation. Moreover, as shown in Merton (1973), the Markowitz-efficient frontier for a market, consisting of portfolios of stocks plus a risk-free security, can be generated by considering portfolio combinations of the NP and the risk-free security. According to the Intertemportal Capital Asset Pricing Model (ICAPM), see Merton (1973), the Sharpe ratios of all portfolios on the efficient frontier are theoretically the same, including that of the market portfolio.

The Naive Diversification Theorem (NDT) is the main theoretical result of this paper. It states that the equi-weighted index (EWI) approximates the NP of a given set of stocks when the number of constituents is large and the given investment universe is well securitized. The latter essentially means that the risk factors driving the underlying risky securities are sufficiently different. The types of funds that resemble the EWI, when used as main investment vehicles for pension funds and insurance companies, would stabilize the market dynamics. It would provide important liquidity in the case when the market crashes. On the other hand, it would sell assets when an asset bubble emerges. In both extreme cases it would serve as an important stabilizing factor in the financial market architecture.

To indicate the power and applicability of the NDT, Fig.1.1 shows over the period from January 1973 until April 2011 an MCI and an EWI in logarithmic scale, each using the same set of stocks, which are those that Datastream reported as 
constituents for its world index in April 2011. The MCI follows the historical market capitalization of all constituent stocks. It can be interpreted as a proxy for the total return market portfolio. Indeed, it fluctuates and performs very similarly to the FTSE all-cap index and the MSCI total return world index. The EWI in Fig. 1.1 takes 40 basis point transaction costs per stock and per trade into account and is constructed according to the description that will be given for the EWI114 with transaction costs as described in Section 5. In the notation of Section 5 the displayed logarithm is that of the EWI114 40 . Even when taking transaction costs into account, the daily reallocated EWI outperforms, in the long-run, the MCI.

This paper interprets the EWI as a good proxy for the NP of the world equity market. It has an average annualized volatility of 0.114 and an estimated annualized expected return of 0.183 . When employing an estimate of 0.05 for the US interest rate, a Sharpe ratio of 1.29 emerges for the EWI. By comparison, the MCI, for which the logarithm is also shown in Fig. 1.1, achieves a Sharpe ratio of 0.537. One notes in Fig. 1.1 that both indices fluctuate very similarly. It is only that the MCI has larger volatility of about 0.134 and a smaller estimated expected return of about 0.108 . This paper documents the existence of such extremely well performing portfolios as the EWI, and attributes their outstanding performance to the diversification effect predicted by the NDT.

The paper is organized as follows: Section 2 describes the construction of diversified indices from real data. Section 3 illustrates theoretically how the Law of Large Numbers drives EWIs towards the NP, as the number of constituents increases. The NDT is formulated and derived in Section 4. Moreover, Section 5 considers the impact of transaction costs on the previously constructed indices, while Section 6 provides a feel for the diversification effect by simulation of naively diversified portfolios for a standard market model.

\section{Diversified Indices}

From an economic point of view it is intuitively appealing that only the nondiversifiable risk should attract a risk premium. This view has been supported by variants of the ICAPM, see e.g. Merton (1973), where the MCI is the source of nondiversifiable risk. This paper systematically employs naive diversification, and captures in this way via the EWI, at least as well as the MCI, nondiversifiable risk. It demonstrates empirically that EWIs perform extremely well, and appear to approach the NP asymptotically, as the number of constituents increases.

To be systematic in capturing the nondiversifiable risk of the global equity market, the paper removes systematically diversifiable risk according to the economic structure of the market. The index constructions performed are based on common types of economic activity, and use accordingly the Industry Classification 
Benchmark (ICB); see ICB (2008). This classification provides a comprehensive structure for the analysis of companies and industries, and has been jointly created by the FTSE and Dow Jones. Four layers of classification for each company are provided. More precisely, the ICB distinguishes 10 industries, 19 supersectors, 41 sectors and 114 subsectors; covering all major industries represented in the world stock market. For instance, industries in the ICB structure include: Oil \& Gas, Basic Materials, Industrials, Consumer Goods, Health Care, Consumer Services, Telecommunications, Utilities, Financials and Technology. The total number of companies covered by the ICB classification system exceeds 60, 000 . Altogether, companies from 72 countries are currently classified. The ICB classifies a company as belonging to a subsector by using the subsector definition that most accurately describes the nature of its business as determined by its largest source of revenue. The paper argues that the ICB classification system is well suited for identifying the nondiversifiable risk generated by the main types of economic activity in the world economy. Note that other similar industry classification systems are likely to provide analogous results. The paper employs the ICB structure as it was used by Thomson Reuters Datastream (Datastream), when evaluating their own industry, supersector, sector and subsector indices.

A representative sample of stocks is chosen by Datastream in each country, for each of the industry subsectors applicable to that country, covering 75 to 80 per cent of the total market capitalization. Moreover, the number of stocks in each subsector is determined by the size of that market sector. The inclusion of a stock by Datastream depends on its total market value and the availability of data. The Datastream subsector and industry indices are updated regularly. This ensures that these indices include the top stocks by market capitalization and reflect new industries and subsectors appropriately. Delisted stocks are removed from an index when notification of a delisting is received. Temporarily suspended stocks remain in an index unless it is believed that the suspension is going to be long term, in which case they are removed from an index. Datastream has a welldefined set of rules that determines the inclusion of stocks in their indices.

The Datastream database is hierarchical and self-contained. For instance, the world Oil \& Gas subsector index was constructed from 52 country Oil \& Gas subsector indices as at the end of April 2011. Furthermore, each country Oil \& Gas index is available together with its constituents. Moreover, the database includes fixed index datatypes and recalculated index datatypes. Fixed index datatypes are not recalculated historically when constituents change, allowing the effects of dead stocks to be observed. The recalculated index datatypes reflect the performance of current constituents, therefore, they avoid any distortion due to stocks entering or leaving an index. Datastream does not provide constituent histories for their sector indices. Consequently, to construct indices that avoid survival bias, the paper uses the fixed datatype, country industry subsector indices as constituents. There are 1969 country industry subsector indices offered by Datastream, which are total return MCIs. When forming comparable MCIs 
and EWIs, the same set of stocks is used, so that the effect of naive diversification can be gauged realistically.

The data are observed daily. Denote by $t_{n}, n \in\{1,2, \ldots\}$, the time of the $n$th observation. Let $S_{t_{n}}^{j, k}$ denote the value of the $j$ th industry, supersector, sector or subsector index for the $k$ th country at time $t_{n}$, as given by Datastream. The number of constituents at the $d$ th classification level, $d \in\{1,10,19,41,114\}$, available in the database equals $\sum_{j=1}^{d} \ell_{d, j}$, where $\ell_{d, j}$ denotes the number of countries for which the $j$ th industry, supersector, sector or subsector was available.

The paper constructs various diversified portfolios, where it applies naive diversification in a systematic way. In a first study it forms five indices $S_{t_{n}}^{\delta_{E W I d}}$, with equally weighted constituents. The value of the equi-weighted index, EWId, having $d$ constituents, $d \in\{1,10,19,41,114\}$, is obtained by the following recursive formula:

$$
S_{t_{n}}^{\delta_{E W I d}}=S_{t_{n-1}}^{\delta_{E W I d}}\left(1+\sum_{j=1}^{d} \sum_{k=1}^{\ell_{d, j}} \pi_{\delta_{E W I d}, t_{n-1}}^{j, k} \frac{S_{t_{n}}^{j, k}-S_{t_{n-1}}^{j, k}}{S_{t_{n-1}}^{j, k}}\right)
$$

with initial value $S_{0}^{\delta_{E W I d}}=100$ and portfolio weights $\pi_{\delta_{E W I d}, t_{n-1}}^{j, k}=\frac{1}{d \ell_{d, j}}, j \in$ $\{1,2, \ldots, d\}, k \in\left\{1,2, \ldots, \ell_{d, j}\right\}$, chosen at time $t_{n-1}$, for those countries which have constituents of this type. Recall that $d \in\{1,10,19,41,114\}$ refers to the number of constituents available at the corresponding classification level according to the ICB structure, and $\ell_{d, j}$ is the corresponding number of countries. Note that the number of constituents in the above indices can change over time. This has been accommodated in the index construction by the use of time dependent fractions.

The resulting family of indices, denominated in US dollars, is shown in Fig. 2.1 for the period from January 1973 until April 2011, and is explained further below. For $d=1$ the EWI1 weights equally the 54 country market capitalization-weighted indices, as provided by Datastream.

For $d=10$, the ICB distinguishes between 10 industries. Here one starts with the market capitalization-weighted country industry indices, as provided by Datastream. For each industry, each country industry index is then equally weighted to yield a corresponding world industry index. Then, by equally weighting the resulting 10 world industry indices, the EWI10 with value $S_{t_{n}}^{\delta_{E W I 10}}$ is obtained.

Analogously, the case $d=19$ considers 19 ICB supersectors and starts with the corresponding market capitalization-weighted country supersector indices. By equally weighting the country supersectors, one obtains world supersector indices, which are then equally weighted to yield the EWI19. Furthermore, the case $d=41$ uses 41 sectors and provides the EWI41. Finally, the case $d=114$ builds on all 114 subsectors, first weighting equally all country subsector indices. It then equally weights the resulting world subsectors to yield the EWI114 with value $S_{t_{n}}^{\delta_{E W I 114}}$. Fig. 2.1 also displays the US dollar value of the MCI, which represents 


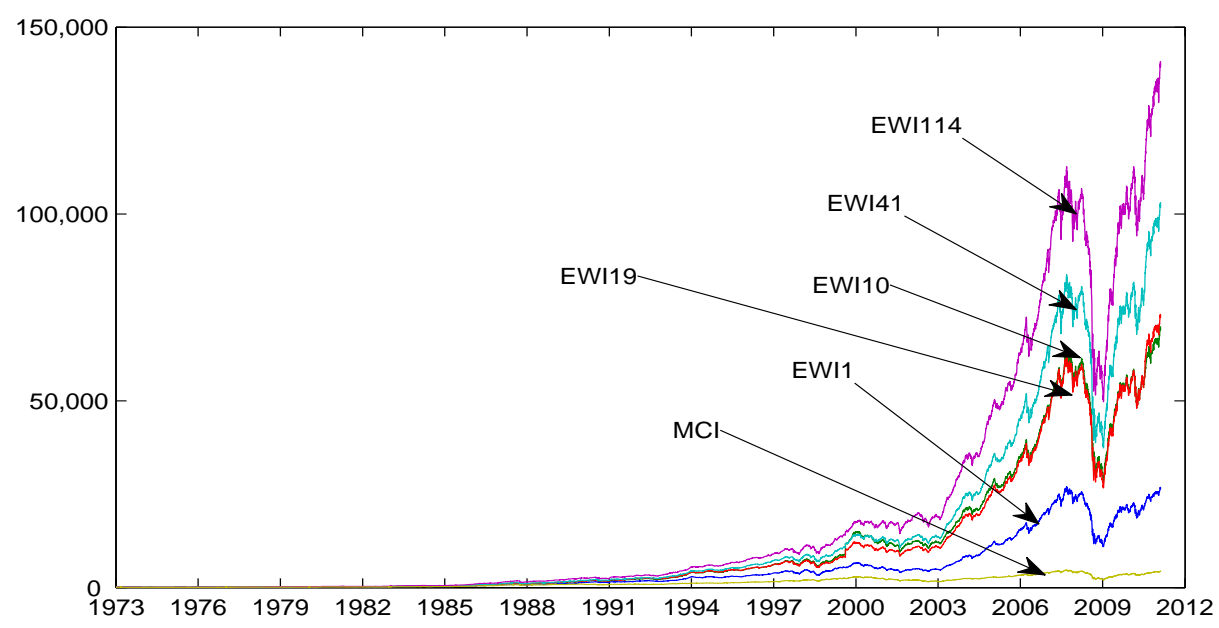

Figure 2.1: The MCI and five equi-weighted indices: EWI1 (market), EWI10 (industry), EWI19 (supersector), EWI41 (sector), EWI114 (subsector).

here the Datastream fixed datatype market capitalization weighted total return world stock index.

In the construction of the MCI the specific risks of large industries and large economies enjoy substantial weight. The MCI performs worst among the indices exhibited in Fig. 2.1. A better display of earlier periods and a visualization of the average long term growth is given by the logarithms of the above described indices, exhibited in Fig. 2.2. One may conjecture that the MCI is exposed to significant diversifiable risks and, therefore, may not yield such a strong average long term growth as some better diversified portfolios. The NDT will later provide theoretical support for such intuition. Among the EWIs displayed in Fig. 2.1, the best performing one is the EWI114, and the worst performing one the EWI1. The EWI10, EWI19 and EWI41 are relatively close to each other. One observes that diversifying with more market capitalization weighted constituents over the same investment universe systematically enhances the average long term growth rate. This is suggesting that smaller fractions achieve visually not only a better diversification effect, but also a higher average long term growth rate. Additionally, one notes from Fig. 2.2 that all EWIs are driven by almost exactly the same continuous uncertainty. This uncertainty can be interpreted as the nondiversifiable risk of the equity market. The MCI is, of course, strongly exposed to this nondiversifiable risk. However, it carries to some extent also the uncertainties of some economies and industries that are heavily weighted by market capitalization. This leads to slightly different fluctuations of the MCI when compared to those of the EWIs, and, as will be explained by the NDT, to lower long term growth. 


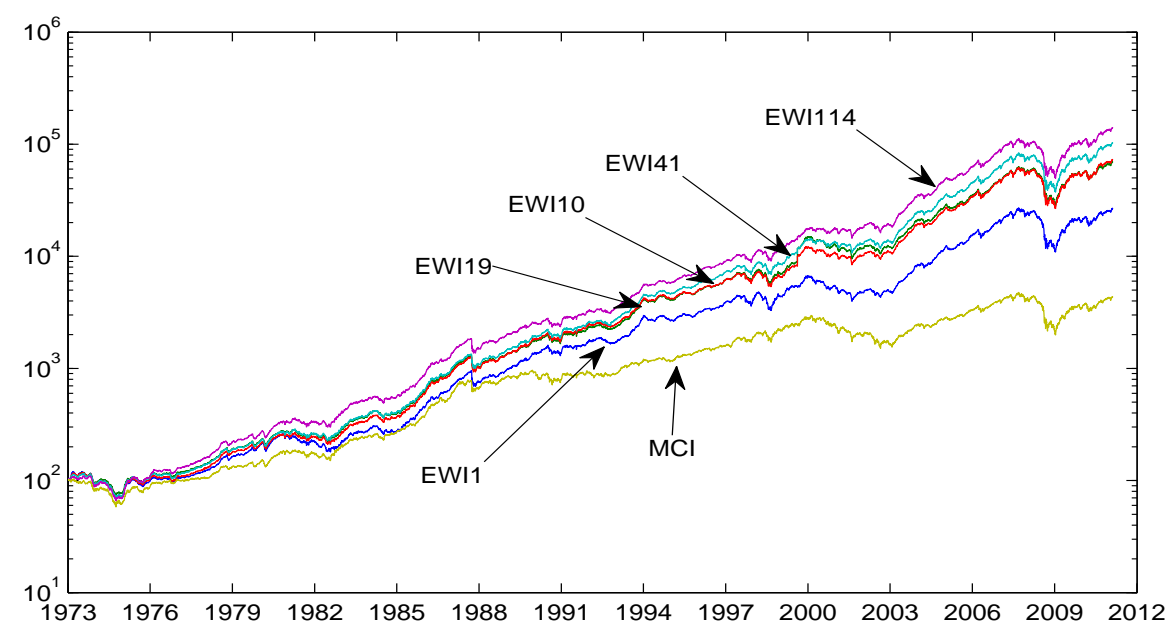

Figure 2.2: The MCI and five equi-weighted indices in log-scale: EWI1 (market), EWI10 (industry), EWI19 (supersector), EWI41 (sector), EWI114 (subsector).

\section{Law of Large Numbers and Diversification}

After having observed that naive diversification works well in practice when focusing on the different types of economic activity, it seems now appropriate to aim for a theoretical understanding of the observed diversification effect. Consider a continuous time market which trades nonnegative primary security accounts. These could represent stocks of companies with all dividends reinvested. In this paper we use total return indicies of industry sectors and other market segments as primary security accounts. As formulated in the introduction, the key assumption of the paper is that there exists a numéraire portfolio (NP). The following arguments employ this strictly positive portfolio as numéraire or benchmark and denominate all primary security accounts in its units. Denote the value of the $j$ th benchmarked primary security account by $\hat{S}_{t}^{j}, j \in\{1,2, \ldots\}$. Since the real market has tens of thousands of stocks, the paper considers an infinite number of benchmarked primary security accounts. This allows the convenient study of the asymptotic behavior of sequences of portfolios with increasing number of constituents.

For a portfolio strategy $\delta=\left\{\delta_{t}=\left(\delta_{t}^{1}, \delta_{t}^{2}, \ldots\right), t \geq 0\right\}$ the quantity $\delta_{t}^{j}$ describes the number of units of the $j$ th benchmarked primary security account held at time $t$. The benchmarked value of this portfolio is given by

$$
\hat{S}_{t}^{\delta}=\sum_{j=1}^{\infty} \delta_{t}^{j} \hat{S}_{t}^{j} .
$$

The paper considers only strategies that are observable under the available information and are such that the corresponding benchmarked portfolio remains 
finite in finite time. Furthermore, the portfolios are assumed to be self-financing, which means that changes in the portfolio value are due only to changes in the values of the primary security accounts.

To introduce for the following the notion of a martingale, denote by $E_{t}(X)$ the conditional expectation of a random variable $X$ under the real-world probability measure $P$, given the information available at time $t$. If the stochastic process $Y=\left\{Y_{t}, t \geq 0\right\}$ satisfies the equation

$$
Y_{t}=E_{t}\left(Y_{s}\right)
$$

for all $s \geq t$, then it is called a martingale, see e.g. Shiryaev (1984). Intuitively, $Y_{t}$ is the best forecast at time $t$ of all future values of the process $Y$.

More generally, a process $Z=\left\{Z_{t}, t \geq 0\right\}$ is called a supermartingale if

$$
Z_{t} \geq E_{t}\left(Z_{s}\right)
$$

for all $s \geq t$; see e.g. Shiryaev (1984) or Platen and Heath (2006) for details and examples. Intuitively, in the long term the forecast of future values of a supermartingale is downward trending or has no trend. In the latter case it is a martingale.

This paper uses the NP as benchmark. A portfolio when denominated in units of the NP is called a benchmarked portfolio. The defining property of the NP is that the values of all benchmarked nonnegative portfolios form supermartingales. This means that the NP performs so well that over any time period the current benchmarked value of any nonnegative portfolio is greater than or equal to any of its expected future benchmarked values. In this simple and specific sense the NP is "best" performing. As in Platen and Heath (2006) one can argue that the above supermartingale property is the central property of a benchmarked portfolio, and several fundamental statements can be directly derived. For example, one can deduce that the path of the NP exceeds asymptotically over time that of any other strictly positive portfolio that starts with the same initial capital; see e.g. Platen and Heath (2006) for a proof of this model independent result.

It should be emphasized that the assumption on the existence of the NP is satisfied for all arbitrage free financial market models. In particular, the condition that a market admits an equivalent risk-neutral probability measure is a stronger assumption.

Given the fact that the NP is in many ways the "best" performing portfolio, it is of great interest from a practical point of view to have direct access to such a tradeable instrument in the global stock market. As indicated in the introduction, the work by DeMiguel, Garlappi and Uppal (2009) has shown that it is unrealistic to hope to identify parameters that estimate trends in asset prices with any useful accuracy using sample-based estimation methods. On the other hand, Section 2 has demonstrated empirically that naively diversified portfolios perform 
extremely well in the long run. The paper will now prove under certain minor technical assumptions that the naive equi-weighted index (EWI) approximates the NP if the number of constituents tends to infinity. This means that there is no need to estimate the parameters of a market model in order to construct a proxy for the NP. Instead, one needs only to construct an EWI with many sufficiently different constituents.

To illustrate the nature of the diversification effect, consider for fixed $t \in[0, \infty)$ and $h>0$ the simple one period case, where the returns $R_{t, h}^{j}=\frac{\hat{S}_{t+h}^{j}-\hat{S}_{t}^{j}}{\hat{S}_{t}^{j}}, j \in$ $\{1,2, \ldots\}$, of the benchmarked primary security accounts $\hat{S}^{1}, \hat{S}^{2}, \ldots$ are independent with zero conditional mean and finite conditional variance. More precisely, assume that

$$
E_{t}\left(R_{t, h}^{j}\right)=0
$$

and

$$
E_{t}\left(R_{t, h}^{j} R_{t, h}^{i}\right)= \begin{cases}\left(\sigma_{t, h}^{j}\right)^{2} & \text { for } j=i \\ 0 & \text { otherwise }\end{cases}
$$

where

$$
\left(\sigma_{t, h}^{j}\right)^{2} \leq \bar{\sigma}_{t, h}^{2}<\infty
$$

can be random. In a classically arbitrage-free continuous market, the zero mean property (3.4) reflects the well-known fact, see Long (1990), that benchmarked primary security accounts are martingales. If the benchmarked primary security accounts have independent uncertainties, due to the different economic activities they model, then the conditions (3.5) and (3.6) are very reasonable.

The return of the benchmarked equi-weighted index $\hat{S}_{t}^{\delta_{E W I \ell}}$, generated by the first $\ell$ benchmarked primary security accounts $\hat{S}_{t}^{1}, \hat{S}_{t}^{2}, \ldots, \hat{S}_{t}^{\ell}$, is

$$
R_{t, h}^{\delta_{E W I \ell}}=\frac{1}{\ell} \sum_{j=1}^{\ell} R_{t, h}^{j}
$$

over the given period. Obviously, by (3.4), one has

$$
E_{t}\left(R_{t, h}^{\delta_{E W I \ell}}\right)=0
$$

Given the independence of the returns of the benchmarked primary security accounts, it follows by (3.5) and (3.6) that

$$
E_{t}\left(\left(R_{t, h}^{\delta_{E W I \ell}}\right)^{2}\right)=\frac{1}{\ell^{2}} \sum_{j=1}^{\ell}\left(\sigma_{t, h}^{j}\right)^{2} \leq \frac{\bar{\sigma}_{t, h}^{2}}{\ell} .
$$

Note that for an increasing number $\ell$ of constituents, the conditional variance of $R_{t, h}^{\delta_{E W I \ell}}$ vanishes asymptotically as $\ell \rightarrow \infty$. Consequently, one obtains zero return for the limiting benchmarked index. Since the benchmarked returns of the NP 
are trivially zero, one notes that the limiting portfolio can only be the NP itself. Below it will become clear that this statement is a particular version of the NDT.

The above arguments rely on the key idea of using the NP as benchmark. Beyond that they are, in principle, an application of the classical Law of Large Numbers. It has been intuitively clear for almost a century that the Law of Large Numbers plays a key role in explaining the effect of diversification. This paper makes this precise by using the conceptual framework of the benchmark approach, see Platen and Heath (2006). The next section removes the above restrictive assumption on the independence of the returns of benchmarked primary security accounts. It is already clear from the above arguments that a wide range of sequences of diversified portfolios approximates the NP. It is the sequence of EWIs which requires minimal assumptions to ensure convergence towards the NP, as will be shown below.

\section{Naive Diversification Theorem}

The Law of Large Numbers is largely model independent and, therefore, very robust. This applies also to the Naive Diversification Theorem (NDT), which will be derived below in general terms. To make the following statements rigorous, stochastic calculus will be applied, which has been widely used in continuous time finance since the work in Merton (1973). In this context the notion of the quadratic variation of a continuous stochastic process $X=\left\{X_{t}, t \geq 0\right\}$ is important. It can be defined as the limit in probability of the sum of squared increments of $X$ when choosing finer and finer equidistant time-discretizations. For instance, the quadratic variation $\langle X\rangle_{t}$ of a standard Brownian motion $X$ equals the time $t$, that is, $\langle X\rangle_{t}=t$. For ease of presentation, this paper focuses on continuous market models. Without any major loss of generality the driving sources of traded uncertainty for benchmarked primary security accounts will be modeled by the Brownian motions $W^{1}, W^{2}, \ldots$

The $j$ th benchmarked primary security account value $\hat{S}_{t}^{j}$ at time $t$ satisfies the driftless Itô stochastic differential equation (SDE)

$$
\frac{d \hat{S}_{t}^{j}}{\hat{S}_{t}^{j}}=\sum_{k=1}^{j} \sigma_{t}^{j, k} d W_{t}^{k} .
$$

One can refer to Merton (1973), Long (1990), Platen and Heath (2006) and Filipović and Platen (2009) for the derivation of such driftless SDEs. Recall from Platen and Heath (2006) that the NP is the growth optimal portfolio which maximizes expected logarithmic utility. It yields in a continuous market the SDE (4.1) when used as numéraire for the $j$ th primary security account. Assume that the volatility processes $\sigma^{j, k}=\left\{\sigma_{t}^{j, k}, t \geq 0\right\}$ are such that the corresponding stochastic integrals in (4.1) exist. Note that the driftless benchmarked primary security accounts are supermartingales. This does not mean that they have 
to be martingales, see Platen and Heath (2006) for details on this issue. The benchmarked self-financing portfolio process $\hat{S}^{\delta}=\left\{\hat{S}_{t}^{\delta}, t \geq 0\right\}$, with strategy $\delta=\left\{\delta_{t}=\left(\delta_{t}^{1}, \delta_{t}^{2}, \ldots\right), t \geq 0\right\}$ is characterized by the SDE

$$
d \hat{S}_{t}^{\delta}=\sum_{j=1}^{\infty} \delta_{t}^{j} d \hat{S}_{t}^{j}
$$

which is driftless. With the introduction of the fraction of wealth invested in the $j$ th primary security account,

$$
\pi_{\delta, t}^{j}=\frac{\delta_{t}^{j} \hat{S}_{t}^{j}}{\hat{S}_{t}^{\delta}}
$$

where $\sum_{j=1}^{\infty} \pi_{\delta, t}^{j}=1$, one can rewrite the $\operatorname{SDE}(4.2)$ in the form

$$
\frac{d \hat{S}_{t}^{\delta}}{\hat{S}_{t}^{\delta}}=\sum_{j=1}^{\infty} \pi_{\delta, t}^{j} \frac{d \hat{S}_{t}^{j}}{\hat{S}_{t}^{j}}=\sum_{j=1}^{\infty} \pi_{\delta, t}^{j} \sum_{k=1}^{j} \sigma_{t}^{j, k} d W_{t}^{k} .
$$

The $\ell$ th equi-weighted index (EWI $\ell$ ) invests the fractions

$$
\pi_{\delta_{E W I \ell}, t}^{j}= \begin{cases}\frac{1}{\ell} & \text { for } j \in\{1,2, \ldots, \ell\} \\ 0 & \text { otherwise. }\end{cases}
$$

Since the benchmarked NP equals the constant one, it follows that there exists a strategy $\delta_{*}=\left\{\delta_{*, t}=\left(\delta_{*, t}^{1}, \delta_{*, t}^{2}, \ldots\right), t \geq 0\right\}$ such that

$$
\frac{d \hat{S}_{t}^{\delta_{*}}}{\hat{S}_{t}^{\delta_{*}}}=\sum_{k=1}^{\infty} \sum_{j=1}^{\infty} \pi_{\delta_{*}, t}^{j} \sigma_{t}^{j, k} d W_{t}^{k}=0 .
$$

This means that the return process of the benchmarked NP equals zero.

It is now the aim to construct sequences of portfolios that approximate the NP in a mathematically precise and practically useful sense. The limits of the return processes of such sequences of benchmarked portfolios should be zero. More precisely, the return process $\hat{Q}^{\delta}=\left\{\hat{Q}_{t}^{\delta}, t \geq 0\right\}$ of a benchmarked portfolio $\hat{S}^{\delta}$, given by the SDE

$$
d \hat{Q}_{t}^{\delta}=\frac{1}{\hat{S}_{t}^{\delta}} d \hat{S}_{t}^{\delta}
$$

for $t \geq 0$ with $\hat{Q}_{0}^{\delta}=0$, has to have small fluctuations to be a good proxy of the NP.

Definition 1 A sequence $\left(\hat{S}^{\delta_{\ell}}\right)_{\ell \in\{1,2, \ldots\}}$ of strictly positive benchmarked portfolios, with initial values equal to one, is called a sequence of benchmarked approximate numéraire portfolios if for each $\varepsilon>0$ and $t \geq 0$ one has

$$
\lim _{\ell \rightarrow \infty} P\left(\frac{d}{d t}\left\langle\hat{Q}^{\delta_{\ell}}\right\rangle_{t}>\varepsilon\right)=0 .
$$


The intuition is that, if one can construct a sequence of benchmarked portfolios where the quadratic variation of the return process vanishes asymptotically, then the limit can only be the constant one, that is, the benchmarked NP.

It seems reasonable to say that the returns of a benchmarked primary security account capture its specific or idiosyncratic traded uncertainty against the market as a whole. Due to the given structure of the market with different types of economic activity in different industry sectors of the economy, it is reasonable to assume that a particular specific uncertainty drives only the returns of a restricted number of benchmarked primary security accounts. If this is the case, then one could say that the securitization of the market is sufficiently developed and a diversification effect can be expected. To capture this property of a market in a mathematically precise manner, one can introduce the following notion:

Definition 2 A financial market is well-securitized if there exists a real number $q>0$ and a stochastic process $\underline{\sigma}^{2}=\left\{\underline{\sigma}_{t}^{2}, t \geq 0\right\}$ with finite mean such that for all $\ell, k \in\{1,2, \ldots\}$ and $t \geq 0$ one has

$$
\frac{1}{\ell}\left|\sum_{j=1}^{\ell} \sigma_{t}^{j, k}\right|^{2} \leq \frac{1}{\ell^{q}} \underline{\sigma}_{t}^{2}
$$

P-almost surely.

Note that for independent benchmarked primary security accounts, as assumed in the previous section, condition (4.9) is easily verified in the presence of finite second moments for the volatility processes. If only a bounded number of benchmarked primary security accounts is driven by the same source of uncertainty, then finite second moments for individual volatilities secure condition (4.9). Even if the number of related returns of benchmarked primary security accounts grows at a rate slower then $\sqrt{\ell}$, then the market still remains well-securitized as long as it has finite second moments for individual volatilities.

We will show below that the proof of the main result of the paper, the NDT, can be based on an even weaker but slightly more technical assumption than formulated in condition (4.9).

Theorem (Naive Diversification Theorem) In a well-securitized financial market the sequence of benchmarked equi-weighted indices, with fractions given by (4.5), is a sequence of benchmarked approximate numéraire portfolios.

We prove this result by using a lemma, which we prepare first. Note that the return process of the $\ell$ th benchmarked EWI has at time $t$ the value

$$
\hat{Q}_{t}^{\delta_{E W I \ell}}=\sum_{j=1}^{\ell} \frac{1}{\ell} \sum_{k=1}^{j} \int_{0}^{t} \sigma_{s}^{j, k} d W_{s}^{k} .
$$


The quadratic variation of this return process is of the form

$$
\left\langle\hat{Q}^{\delta_{E W I \ell}}\right\rangle_{t}=\frac{1}{\ell} \int_{0}^{t} \sum_{k=1}^{\ell}\left|\frac{1}{\sqrt{\ell}} \sum_{j=1}^{\ell} \sigma_{s}^{j, k}\right|^{2} d s .
$$

As can be seen below, this particular structure allows one to use a weaker slightly more technical assumption than imposed in (4.9) to guarantee a sequence of benchmarked approximate numéraire portfolios.

Lemma 1 Assume that for all $\varepsilon>0$ and $t \geq 0$ one has the limit in probability

$$
\lim _{\ell \rightarrow \infty} P\left(\frac{1}{\ell} \sum_{k=1}^{\ell}\left|\frac{1}{\sqrt{\ell}} \sum_{j=1}^{\ell} \sigma_{t}^{j, k}\right|^{2}>\varepsilon\right)=0,
$$

then the sequence of equi-weighted indices is a sequence of benchmarked approximate numéraire portfolios.

\section{Proof of Lemma 1:}

The statement of the above lemma is obtained via (4.8) in Definition 1, together with (4.11) and (4.12) since for $\varepsilon>0$ and $t>0$ one obtains directly that

$$
\lim _{\ell \rightarrow \infty} P\left(\frac{d}{d t}\left\langle\hat{Q}^{\delta_{E W I \ell}}\right\rangle_{t}>\varepsilon\right)=\lim _{\ell \rightarrow \infty} P\left(\frac{1}{\ell} \sum_{k=1}^{\ell}\left|\frac{1}{\sqrt{\ell}} \sum_{j=1}^{\ell} \sigma_{t}^{j, k}\right|^{2}>\epsilon\right)=0 .
$$

\section{Proof of Naive Diversification Theorem:}

It is straightforward to see by the well-known Markov inequality that in a wellsecuritized market the assumption (4.12) is satisfied due to condition (4.9) since

$$
\lim _{\ell \rightarrow \infty} P\left(\frac{1}{\ell} \sum_{k=1}^{\ell}\left|\frac{1}{\sqrt{\ell}} \sum_{j=1}^{\ell} \sigma_{t}^{j, k}\right|^{2}>\varepsilon\right)=\lim _{\ell \rightarrow \infty} P\left(\frac{1}{\ell^{q}} \underline{\sigma}_{t}^{2}>\varepsilon\right) \leq \lim _{\ell \rightarrow \infty} \frac{1}{\ell^{q}} \frac{1}{\varepsilon} E\left(\underline{\sigma}_{t}^{2}\right)=0,
$$

which proves the Naive Diversification Theorem.

Note from the above that if condition (4.12) does not hold, then also the statement (4.13) cannot be true, which shows that in this case naive diversification does not lead to convergence in probability towards the NP. The statement of the NDT is very robust. Already, under condition (4.12), which is weaker than (4.9), it covers a wide range of models. Essentially, one can say that the NDT is model independent since no particular assumptions about the underlying market model need to be made. We emphasize, the NDT, and more generally the above Lemma 4.1, are based on very few probabilistic arguments, and no major economic assumptions have to be made. The presence of a large number of 


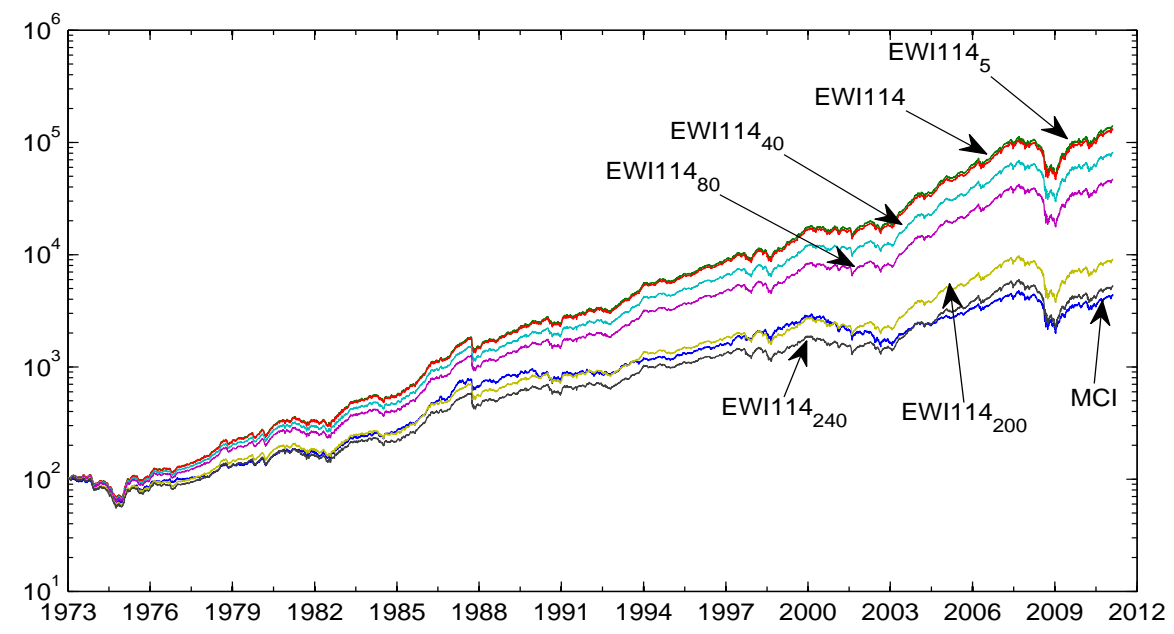

Figure 5.1: Logarithms of MCI, EWI114 without transaction cost and EWI114 $4_{\xi}$ with transaction costs of $5,40,80,200$ and 240 basis points.

reasonably different economic activities securitized in the market is sufficient to let the sequence of EWIs automatically approximate the NP. This provides another view to economic modeling. Simply the laws of statistics provide the fact that a naively diversified portfolio is a good proxy of the NP, which forms naturally the central building block of the benchmark approach. The latter, however, represents a generalization of the classical arbitrage pricing theory and modern portfolio theory, see Platen and Heath (2006)

\section{Transaction Costs and Reallocation Frequency}

This section demonstrates that the impact of transaction costs is almost negligible from the perspective of large funds. The observed long term growth of the EWI114 is, in principle, obtainable by an investable portfolio. Additionally, the influence of the length of the time between capital reallocation is studied in this section. Only minor changes in performance are observed for the cases with time periods between capital reallocations of up to one month.

In the case when constructing equi-weighted indices it is necessary to reallocate regularly capital according to the naive diversification strategy. Naive diversification constitutes a form of hedging. To compare realistically the long term performance of portfolios one needs to account for transaction costs incurred by reallocations. That is, the value of an EWI at each reallocation has to be reduced by the respective transaction cost. When forming the equi-weighted index $S^{\delta_{E W I_{\xi}}}$ in US dollars, the study assumes proportional transaction costs $\xi$ to be charged 


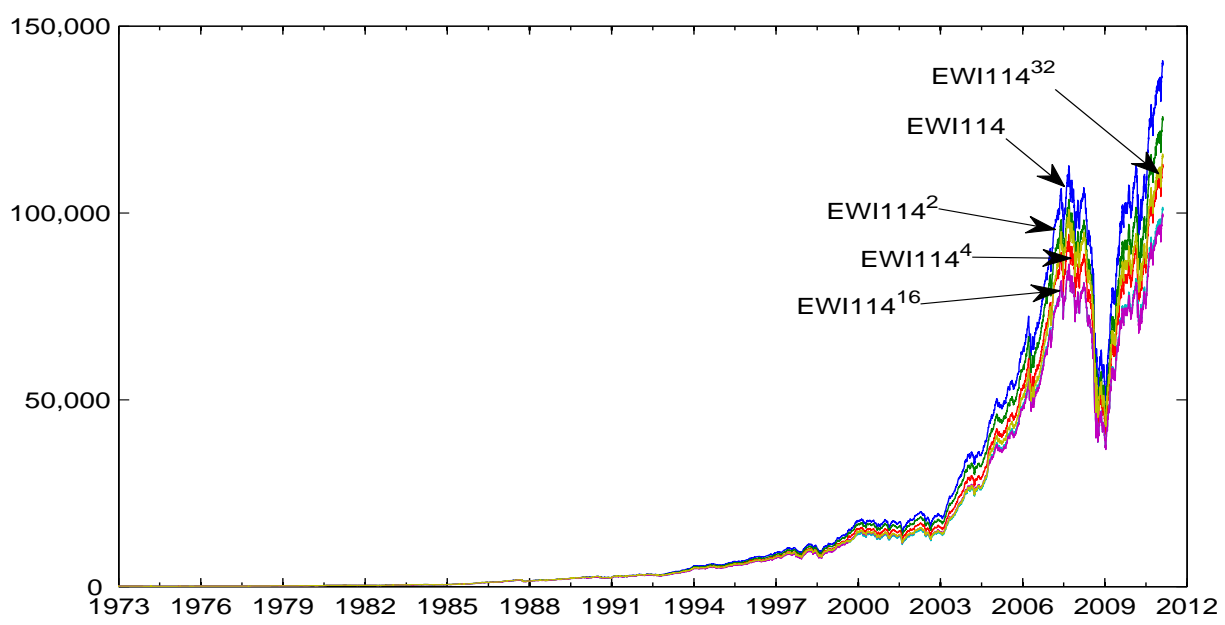

Figure 5.2: EWI114 ${ }^{m}$ reallocated daily and every 2, 4, 8, 16 and 32 days.

in the following way:

$$
S_{t_{n}}^{\delta_{E W I_{\xi}}}=S_{t_{n}-}^{\delta_{E W I_{\xi}}}-\sum_{j=1}^{d} \frac{\xi}{10000} S_{t_{n}}^{j}\left|\delta_{E W I_{\xi}, t_{n}}^{j}-\delta_{E W I_{\xi}, t_{n-1}}^{j}\right|,
$$

where the number $d$ of constituents is partly suppressed in the notation, and $\xi$ denotes the parameter for the proportional transaction cost in basis points (bp). Note that one has $\delta_{E W I_{\xi}, t_{n}}^{j}=\frac{1}{d}$ in (5.1). In the above formula $S_{t_{n}}^{j}$ denotes the value of the $j$ th primary security account in US dollars at time $t_{n}$. Furthermore, $S_{t_{n}-}^{\delta_{E W I_{\xi}}}$ denotes the value of the EWI in US dollars just before reallocation takes place at time $t_{n}$, whereas $S_{t_{n}}^{\delta_{E W I_{\xi}}}$ equals its value after reallocation and after accounting for transaction costs. Note that the results are, in principle, the same if one uses another base currency.

This section studies only indices constructed similarly to the EWI114 but with different values for the transaction cost parameter $\xi$. Later different time intervals between capital reallocations will be allowed. In general, one expects larger transaction costs to yield lower average long term growth. Indeed, this can be observed in Fig. 5.1, which displays the logarithms of the MCI, the EWI114 and the EWI114 $\xi$, constructed for the transaction cost parameter values $\xi \in\{5,40,80,200,240\}$ under daily capital reallocation. The EWI $114_{5}$ has only marginally lower values than the EWI114 and both are almost indistinguishable. In the long run the MCI is located approximately between the EWI114 200 and the EWI114 $2_{24}$, suggesting that it takes roughly between 200 and $240 \mathrm{bp}$ in proportional transaction costs to bring the long term growth of the EWI114 $4_{\xi}$ down to that of the MCI when reallocating daily. Transaction costs of $200 \mathrm{bp}$ are significantly beyond those charged to large funds. Note in Fig. 5.1 that all EWIs are driven by almost exactly the same nondiversifiable risk factor. The MCI is 
Table 1: Summary for various EWI114 versions with transaction cost equal to $\xi \in\{0,5,40,80,200,240\}$ basis points

\begin{tabular}{|c|c|c|c|c|c|c|}
\hline Transaction cost & 0 & 5 & 40 & 80 & 200 & 240 \\
\hline $\begin{array}{l}\text { Reallocation terms } \\
\text { Final value } \\
\text { Annualised average return } \\
\text { Annualised volatility } \\
\text { Sharpe ratio }\end{array}$ & $\begin{array}{r}1 \\
139338.64 \\
0.1979 \\
0.1135 \\
1.4205\end{array}$ & $\begin{array}{r}130111.93 \\
0.1961 \\
0.1135 \\
1.4046\end{array}$ & $\begin{array}{r}80543.07 \\
0.1834 \\
0.1135 \\
1.2930\end{array}$ & $\begin{array}{r}46555.04 \\
0.1689 \\
0.1135 \\
1.1654\end{array}$ & $\begin{array}{r}8988.23 \\
0.1254 \\
0.1134 \\
0.7822\end{array}$ & $\begin{array}{r}5194.46 \\
0.1109 \\
0.1134 \\
0.6544\end{array}$ \\
\hline $\begin{array}{l}\text { Reallocation terms } \\
\text { Final value } \\
\text { Annualised average return } \\
\text { Annualised volatility } \\
\text { Sharpe ratio }\end{array}$ & $\begin{array}{r}2 \\
124542.04 \\
0.1949 \\
0.1134 \\
1.3955\end{array}$ & $\begin{array}{r}119369.00 \\
0.1938 \\
0.1134 \\
1.3856\end{array}$ & $\begin{array}{r}88697.63 \\
0.1859 \\
0.1134 \\
1.3163\end{array}$ & $\begin{array}{r}63166.73 \\
0.1770 \\
0.1134 \\
1.2369\end{array}$ & $\begin{array}{r}22808.64 \\
0.1500 \\
0.1135 \\
0.9987\end{array}$ & $\begin{array}{r}16240.40 \\
0.1411 \\
0.1136 \\
0.9193\end{array}$ \\
\hline $\begin{array}{l}\text { Reallocation terms } \\
\text { Final value } \\
\text { Annualised average return } \\
\text { Annualised volatility } \\
\text { Sharpe ratio }\end{array}$ & $\begin{array}{r}4 \\
111899.82 \\
0.1921 \\
0.1135 \\
1.3699\end{array}$ & $\begin{array}{r}108230.16 \\
0.1912 \\
0.1135 \\
1.3622\end{array}$ & $\begin{array}{r}85698.25 \\
0.1850 \\
0.1134 \\
1.3080\end{array}$ & $\begin{array}{r}65628.82 \\
0.1780 \\
0.1134 \\
1.2459\end{array}$ & $\begin{array}{r}29467.48 \\
0.1568 \\
0.1134 \\
1.0591\end{array}$ & $\begin{array}{r}22562.42 \\
0.1497 \\
0.1135 \\
0.9967\end{array}$ \\
\hline $\begin{array}{l}\text { Reallocation terms } \\
\text { Final value } \\
\text { Annualised average return } \\
\text { Annualised volatility } \\
\text { Sharpe ratio }\end{array}$ & $\begin{array}{r}8 \\
100505.37 \\
0.1892 \\
0.1127 \\
1.3531\end{array}$ & $\begin{array}{r}97963.66 \\
0.1885 \\
0.1127 \\
1.3471\end{array}$ & $\begin{array}{r}81881.57 \\
0.1837 \\
0.1127 \\
1.3051\end{array}$ & $\begin{array}{r}66705.62 \\
0.1783 \\
0.1127 \\
1.2569\end{array}$ & $\begin{array}{r}36055.10 \\
0.1621 \\
0.1128 \\
1.1119\end{array}$ & $\begin{array}{r}29367.02 \\
0.1566 \\
0.1128 \\
1.0634\end{array}$ \\
\hline $\begin{array}{l}\text { Reallocation terms } \\
\text { Final value } \\
\text { Annualised average return } \\
\text { Annualised volatility } \\
\text { Sharpe ratio }\end{array}$ & $\begin{array}{r}16 \\
98775.24 \\
0.1887 \\
0.1130 \\
1.3463\end{array}$ & $\begin{array}{r}96892.29 \\
0.1882 \\
0.1130 \\
1.3418\end{array}$ & $\begin{array}{r}84677.43 \\
0.1847 \\
0.1130 \\
1.3102\end{array}$ & $\begin{array}{r}72588.14 \\
0.1806 \\
0.1130 \\
1.2740\end{array}$ & $\begin{array}{r}45711.97 \\
0.1684 \\
0.1131 \\
1.1647\end{array}$ & $\begin{array}{r}39177.91 \\
0.1643 \\
0.1131 \\
1.1281\end{array}$ \\
\hline $\begin{array}{l}\text { Reallocation terms } \\
\text { Final value } \\
\text { Annualised average return } \\
\text { Annualised volatility } \\
\text { Sharpe ratio }\end{array}$ & $\begin{array}{r}32 \\
114592.50 \\
0.1927 \\
0.1131 \\
1.3797\end{array}$ & $\begin{array}{r}112929.09 \\
0.1923 \\
0.1131 \\
1.3763\end{array}$ & $\begin{array}{r}101939.59 \\
0.1896 \\
0.1131 \\
1.3522\end{array}$ & $\begin{array}{r}90678.85 \\
0.1865 \\
0.1131 \\
1.3245\end{array}$ & $\begin{array}{r}63804.84 \\
0.1772 \\
0.1133 \\
1.2408\end{array}$ & $\begin{array}{r}56744.28 \\
0.1741 \\
0.1133 \\
1.2127\end{array}$ \\
\hline
\end{tabular}

exposed to some additional risk factors since it does not always move perfectly parallel to the EWIs.

Let us now study, first without transaction costs, the resulting EWIs when reallocating every 2,4,8,16 and 32 days. Fig. 5.2 displays these indices together with the EWI114. Denote by EWI114 ${ }^{m}$ the index which reallocates every $m$ days without transaction costs. The EWI $114^{m}$ does not outperform the daily reallocated EWI114, for $m \in\{2,4,8,16,32\}$. However, if the reallocations are less frequent, then by chance occasional outperformance of the EWI114 by such constructed indices can be expected. The NDT predicts that in the long-run the EWIs with sufficiently frequent capital reallocations should approximate well the NP and, thus, outperform other indices.

Below, the paper studies the combined impact of different transaction cost parameters and different time intervals between reallocations. Table 1 displays the final value of the respective indices, the estimated annualized expected return $\hat{\mu}$, the estimated annualized volatility $\hat{\sigma}$ and the resulting Sharpe ratio $\hat{s}=\frac{\hat{\mu}-\hat{r}}{\hat{\sigma}}$. 


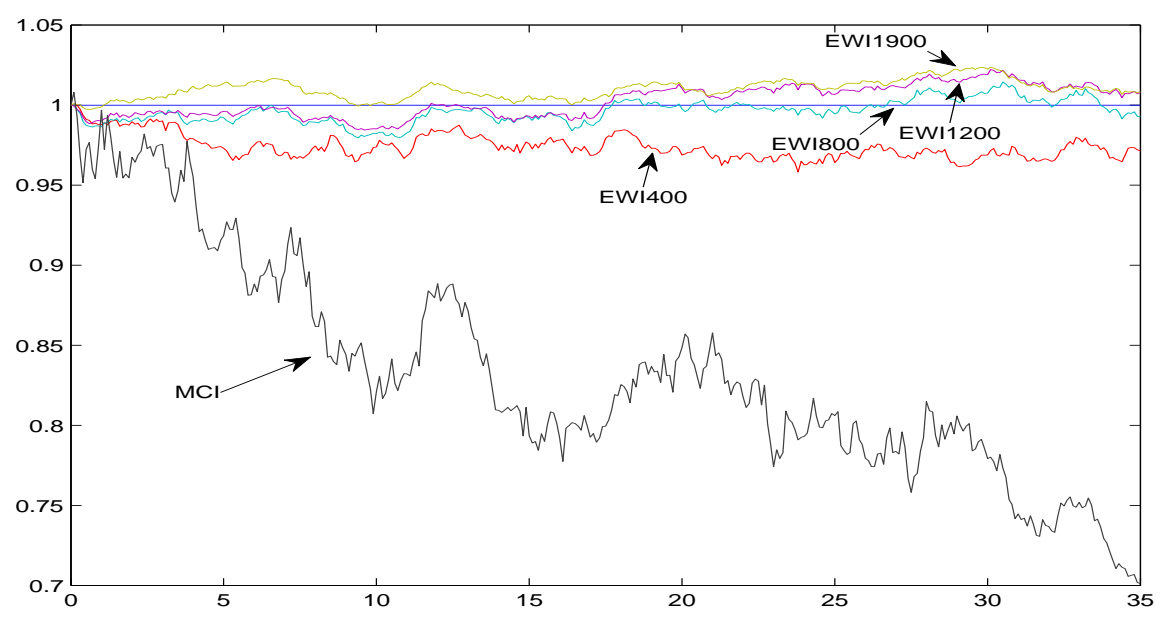

Figure 6.1: Simulated benchmarked MCI and EWIs.

The Sharpe ratio is here evaluated as the ratio of the difference between the annualized sample mean of the returns minus an assumed average US short rate $\hat{r}$ of 0.05 , over the annualized sample standard deviation $\hat{\sigma}$. Recall that the MCI, mentioned in Section 1, has the estimated volatility of 0.134 and the estimated expected return of 0.108 , which yields a Sharpe ratio of 0.537 .

The results for the indices constructed with different days $m \in\{1,2,4,8,16,32\}$ between capital reallocations and with transaction cost parameter $\xi \in\{0,5$, $40,80,200,240\}$ are displayed in Table 1 . In this table the estimated volatilities remain very close to each other up to quarterly reallocations. With an annualized value of approximately 0.11 they are clearly smaller than the estimated volatility of 0.13 for the MCI. The largest Sharpe ratio reported in Table 1 with value 1.42 is that of the EWI114 with daily reallocations and without transaction costs. The Sharpe ratio of the EWI114 ${ }^{m}$ seems to decline for increasing $m \in\{1,2,4,8,16\}$. Even with very realistic transaction costs of $80 \mathrm{bp}$ the Sharpe ratios of the different equi-weighted indices remain in this table above twice the Sharpe ratio of the MCI. In summary, we see in Table 1 that less frequent reallocations and higher transaction costs diminish, in general, the Sharpe ratio.

\section{Simulation of a Standard Market Model}

To provide a feeling for the impact of the diversification effect this brief section illustrates the asymptotic properties of simulated naively diversified portfolios. It simulates over a period of 35 years under a Black-Scholes market model $d=$ 1900 independent, driftless, benchmarked primary security accounts, each with constant volatility 0.15 . It also simulates random initial values of the stocks, 
distributed according to a Pareto distribution with Pareto parameter $\alpha=1.11$ and mean one. The Pareto parameter $\alpha$ is chosen similarly to the one estimated in Simon and Bonini (1958) for the distribution of company sizes. The assumed Pareto distribution aims to give a reasonably realistic reflection of typical market capitalizations of stocks in this simulation study, which forms four equi-weighted indices, the EWI400, EWI800, EWI1200 and EWI1900, by using the first $d=400$, 800,1200 and 1900 constituents, respectively. The trajectories of the resulting benchmarked portfolios are shown in Fig. 6.1. The benchmarked NP equals the constant one. As suggested by the NDT, a benchmarked good proxy of the NP should come close to the constant one.

After the MCI, the EWI400 shows in Fig. 6.1 the largest discrepancy, whereas the EWI1900 comes rather close to the benchmarked NP. When repeating this simulation for other scenarios the outcomes appear to be similar.

The stocks with the largest average fraction are likely to contribute most to the deviation of the benchmarked MCI from the benchmarked NP, the constant level one, in Fig. 6.1. Note that such deviation can go for a while in either direction. In the long run, however, it is always the NP that outperforms with its path that of any other portfolio.

In summary, the equi-weighted index with the largest number of constituents approximated best the NP in this simulation study. This is consistent with the prediction of the NDT and observations on real data. By performing a simulation of the above kind with given realistic market dynamics one can obtain a valuable impression about how strong the predicted diversification effect may be in reality. In Platen and Rendek (2010), the authors perform a detailed simulation study on the effect of diversification for a range of market models beyond the standard market model.

\section{Conclusion}

This paper aims to give a better understanding of the basic principle underpinning the well-observed phenomenon of diversification, fundamental to fund and risk management. It approximates the numéraire portfolio, which is in many ways the "best" performing portfolio, by a sequence of naive (equi-weighted) portfolios with increasing number of constituents. The Naive Diversification Theorem has been established, which states that a naively diversified portfolio with many constituents is a good proxy for the numéraire portfolio of a well-securitized market. By applying naive diversification on real equity data, the paper identifies an equi-weighted index, formed by equi-weighted country subsector indices, as a good proxy of the numéraire portfolio of the global stock market. This proxy turns out to be the best performing index in the family of diversified portfolios constructed in this paper. Its outstanding long term performance has been predicted by the 
Naive Diversification Theorem. An equi-weighted index should show an excellent average long term growth rate due to its proximity to the numéraire portfolio. The paper demonstrates that naive diversification resolves practical difficulties encountered with the sample-based Markowitz mean-variance approach to portfolio optimization. There is no need for estimating drift parameters. Even if one accounts for transaction costs in the construction of an equi-weighted index, it still shows a significantly higher Sharpe ratio when compared to the one of the corresponding market capitalization weighted index. The proposed approach of approximating the numéraire portfolio by naive diversification is robust and also very general. With the possible construction of an investable proxy for the numéraire portfolio, this paper opens new lines of research. The numéraire portfolio is not only in several ways the "best" performing portfolio, it is also the natural numéraire for derivative pricing under the real-world probability measure. Furthermore, as a consequence of the Naive Diversification Theorem it captures the nondiversiable risk of the market. This general market risk is highly relevant for risk measurement and risk management. Moreover, when equi-weighted world indices of the proposed type would become major investment vehicles in the financial market architecture used by large pension funds and insurance companies, they would have a stabilizing effect. In the time of a financial crisis they would provide liquidity to those asset classes under stress. On the other hand, in the case of an asset bubble they would moderate the excesses by selling highly priced assets. This goes along with the intuitive reasoning behind naive diversification, which always sells high and buys low.

\section{Acknowledgement}

The Industry Classification Benchmark system ICB has been applied, jointly developed by the FTSE and Dow Jones. The data utilized was provided by Thomson Reuters Datastream. The authors like to thank Harry Markowitz, Stephen Satchell and Adrian Pagan for their interest and stimulating discussions on diversified portfolios, as well as, Hardy Hulley, Vikram Kuriyan and Anthony Tooman for suggestions on the paper.

\section{References}

Bajeux-Besnainou, I. and Portait, R. (1997) The numeraire portfolio: A new perspective on financial theory. The European Journal of Finance 3: 291309.

Becherer, D. (2001) The numeraire portfolio for unbounded semimartingales. Finance Stoch. 5: 327-341.

Best, M. J. and Grauer, R. R. (1991) On the sensitivity of mean-varianceefficient portfolios to changes in asset means: Some analytical and compu- 
tational results. Rev. Financial Studies 4(2): 315-342.

Breiman, L. (1961) Optimal gambling systems for favorable games. In: Proceedings of the Fourth Berkeley Symposium on Mathematical Statistics and Probability, Volume I, pp. 65-78.

Browne, S. (1999) The risks and rewards of minimizing shortfall probability. J. Portfolio Manag. 25(4): 76-85.

Bühlmann, H. and Platen, E. (2003) A discrete time benchmark approach for insurance and finance. Astin Bulletin 33(2): 153-172.

Campbell, J. and Viceira, L. (2002) Strategic Asset Allocation, Portfolio Choice for Long Term Investors. Oxford: Oxford University Press.

Cover, T. (1991) Universal portfolios. Math. Finance 1: 1-29.

DeMiguel, V., Garlappi, L. and Uppal, R. (2009) Optimal versus naive diversification: How inefficient is the $1 / n$ portfolio strategy? Rev. Financial Studies 22(5): 1915-1953.

Filipović, D. and Platen, E. (2009) Consistent market extensions under the benchmark approach. Math. Finance 19(1): 41-52.

Hakansson, N. H. (1971) Capital growth and the mean-variance approach to portfolio selection. J. Financial and Quantitative Analysis 6(1): 517-557.

Hofmann, N. and Platen, E. (2000) Approximating large diversified portfolios. Math. Finance 10(1): 77-88.

ICB (2008). Industry classification benchmark. http://rcbenchmark.com/index.html, accessed 5 March 2011.

Karatzas, I. and Kardaras, C. (2007) The numeraire portfolio in semimartingale financial models. Finance Stoch. 11(4): 447-493.

Kardaras, C. and Platen, E. (2008) On financial markets where only buyand-hold trading is possible. Sydney, Australa: University of Technology, Sydney. QFRC Research Paper no. 213.

Kelly, J. R. (1956) A new interpretation of information rate. Bell Syst. Techn. J. 35: 917-926.

Latané, H. (1959) Criteria for choice among risky ventures. J. Political Economy 38: $145-155$.

Litterman, B. and the Quantitative Research Group (2003) Modern Investment Management: An Equilibrium Approach. Wiley Finance.

Long, J. B. (1990) The numeraire portfolio. J. Financial Economics 26: 29-69.

Luenberger, D. G. (1998) Investment Science. Oxford University Press.

MacLean, L.C. and Thorp, E. O. and Ziemba, W. (2011) The Kelly Capital Growth Investment Criterion. World Scientific.

Markowitz, H. (1952) Portfolio selection. J. Finance VII(1): 77-91. 
Markowitz, H. (1976) Investment for the long run: New evidence for an old rule. J. Finance XXXI(5): 1273-1286.

Merton, R. C. (1973) An intertemporal capital asset pricing model. Econometrica 41: 867-888.

Platen, E. (2002) Arbitrage in continuous complete markets. Adv. in Appl. Probab. 34(3): 540-558.

Platen, E. (2004) A benchmark framework for risk management. In: Stochastic Processes and Applications to Mathematical Finance, pp. 305-335. Proceedings of the Ritsumeikan Intern. Symposium: World Scientific.

Platen, E. (2005) Diversified portfolios with jumps in a benchmark framework. Asia-Pacific Financial Markets 11(1): 1-22.

Platen, E. (2006) A benchmark approach to asset management. J. Asset Management 6(6): 390-405.

Platen, E. and Heath, D. (2006) A Benchmark Approach to Quantitative Finance. Springer Finance. Springer.

Platen, E. and Rendek, R. (2010) Simulation of diversified portfolios in a continuous financial market. Sydney, Australia: University of Technology, Sydney. QFRC Research Paper no. 264.

Rubinstein, M. (1976) The strong case for the generalized logarithmic utility model as the premier model of financial markets. J. Finance 31: 551-571.

Shiryaev, A. N. (1984) Probability. Springer.

Simon, H. A. and Bonini, C. P. (1958) The size distribution of business firms. Amer. Econ. Rev. 48(4): 607-617.

Stutzer, M. J. (2000) A portfolio performance index. Financial Analysts J. $56(3): 52-61$.

Thorp, E. O. (1972) Portfolio choice and the Kelly criterion. In: Proceedings of the $19^{r} 1$ Business and Economics Section of the American Statistical Association, Volume 21, pp. 5-224.

Ziemba, W. T. and Mulvey, J. M. (1998) Worldwide Asset and Liability Modeling. Cambridge University Press. 